\title{
Nurses' movements within and between various paths when improving their communication skills-An evaluation of validation method training
}

\author{
Mona Söderlund $^{1,2}$, Agneta Cronqvist ${ }^{1}$, Astrid Norberg ${ }^{1,3,4}$, Britt-Marie Ternestedt ${ }^{1,2,3,5}$, \\ Görel Hansebo ${ }^{1,2}$ \\ ${ }^{1}$ Department of Health Care Sciences, Ersta Sköndal University College, Stockholm, Sweden \\ ${ }^{2}$ Department of Neurobiology, Care Sciences and Society, Karolinska Institutet, Stockholm, Sweden \\ ${ }^{3}$ Palliative Research Centre, Ersta Sköndal University College \& Ersta Hospital, Stockholm, Sweden \\ ${ }^{4}$ Department of Nursing, Umeå University, Umeå, Sweden \\ ${ }^{5}$ Stockholm Sjukhem Foundation, Research and Development Department, Stockholm, Sweden \\ Email: mona.soderlund@esh.se
}

Received 27 February 2013; revised 28 March 2013; accepted 15 April 2013

Copyright (C) 2013 Mona Söderlund et al. This is an open access article distributed under the Creative Commons Attribution License, which permits unrestricted use, distribution, and reproduction in any medium, provided the original work is properly cited.

\section{ABSTRACT}

Aims and objectives: To explore any changes in nurses' skills at communicating with residents with dementia disease when using the validation method, as observed in one-to-one videotaped conversations. Background: Communication difficulties due to cognitive impairment among residents with dementia disease may complicate care situations. Training can improve nurses' communication skills and increase care quality. The validation method aims to facilitate communication with residents with dementia disease through empathic and confirmatory approaches. Evaluations of the validation method have primarily focused on the residents' perspective, and reports on nurses' experiences are sparse. Improved communication and relationships with residents after validation method training have been described previously. Videotaped data could provide additional information about these earlier results. Design: A descriptive qualitative design. Methods: Eight nurses participated in a year of validation method training, including videotaped conversations with eleven residents. Videotapes with at least five months between the first and last recording were analysed and compared qualitatively. Results: The analysis revealed an overall pattern: nurses' movements within and between various paths when improving their communication skills. This was based on three sub-patterns: from controlling communication towards developing attentiveness in communication, from ambiguous communication towards developing coherence in communication, and from being open and attentive towards having a refined attuned communication. Conclusions: All nurses developed their communication skills during the programme, albeit to different degrees. The findings are in congruence with the experiences described by nurses, and so it is reasonable to believe that the programme helped to improve the nurses' skills in communicating with residents with dementia disease. Relevance to clinical practice: A validation method training programme could give nurses the possibility to develop their skills in communicating with residents with dementia disease.

Keywords: Training Communication Skills; Dementia Care; Validation Method; Qualitative Analysis of Videotapes

\section{INTRODUCTION}

Many challenging caring situations can arise in everyday care of residents with dementia disease (DD) living in nursing homes, due to these residents' difficulties in expressing their needs and wishes. The cognitive impairments that accompany DD affect the ability to communicate; this can cause discomfort, anxiety, or agitation, which has been reported to occur among $74 \%$ of the residents in a nursing home [1]. This can lead to challenging caring situations that may be experienced as frightening by nurses, and may also create uncertainty in how to approach residents.

Nurses working in dementia care have expressed difficulties both in understanding what residents are trying 
to communicate and in making themselves understood [2]. The reasons behind this may include insufficient knowledge about the disease and how it affects communication, as well as a lack of engagement and interest in dementia care. Residents' need of and ability to communicate may be underestimated as a consequence [3]. Therefore, it is of critical importance for the wellbeing of residents that all persons involved in their care, regardless of occupation, are skilled in communicating with them [4].

Review studies have shown that training nurses in communication with residents with DD may increase care quality and be beneficial for both residents and nurses $[5,6]$. Although the training programmes in these reviews differed in duration, intervention components, and teaching strategies, the results demonstrate that the communication training changed nurses' skills to become warmer, more personal, and empathetic in the communication with residents.

The present study is part of a larger project involving implementation of the validation method (VM) in nursing home wards, described in detail elsewhere [7]. The VM is an empathetic and confirmatory approach that includes accepting a person's experiences of reality to facilitate communication [8]. The VM strives for responsiveness to residents' verbally and/or non-verbally expressed actions and reactions, by use of specific VM techniques for communication (see Appendix 1). The VM is intended for use in both one-to-one conversations and group conversations with disoriented persons with DD around the age of 80 and without a medical history of psychiatric illness or alcohol abuse [8].

Research conducted to evaluate the VM has primarily focused on group conversations and the perspective of the residents with DD, and a Cochrane review suggests that there is insufficient scientific evidence for the effects of the VM [9]. Reports on nurses' experiences of the one-to-one VM conversations are sparse. In a study with a quasi-experimental design, Fine and Rouse-Bane [10] observed interactions between nurses and residents before and after VM training, and reported that after VM training the nurses responded more effectively to the needs and behaviours expressed by residents. Finnema $e t$ $a l$. [11] used a randomised controlled trial design and reported fewer stress reactions among nurses who used emotion-oriented care skills, including the VM. Söderlund et al. [7] used a qualitative design and reported that nurses described improved communication and closer relationships with residents after participating in a VM training programme with supervision. However, the participating nurses also described difficulties with using the VM, and said that it was demanding. One question that should be asked is whether these nurses' actions are congruent with their expressed improvements in their relationships with residents. Therefore it seemed necessary to study the nurses' communications with residents that had been videotaped during the VM training programme. These data could provide additional information about the nurses' expressed improvements in communication skills.

\section{AIM}

The aim of the present study was to explore any changes in nurses' communication skills with residents with dementia disease when using the validation method, as observed in videotaped one-to-one conversations.

\section{METHODS}

This study had a descriptive qualitative design and the focus was on the nurses' communications. All participants are referred to as "nurses" here, regardless of whether they were registered nurses, licensed practical nurses, or nurses' aides.

\subsection{Study Background}

A one-year VM training programme (hereafter referred to as "the programme") was implemented in three nursing home wards in a large Swedish city. The nursing home had traditional wards each including 24 - 27 residents divided into groups of 8 - 9 residents. The nurses worked in care teams, with each nurse having a care responsibility for 1 - 2 residents. The programme was led by an external certified supervisor in line with the guidelines of the Validation Training Institute [12]. It was directed towards a group of nurses, regardless of professsion, and included 10 days of theoretical VM training with supervision about once a month. During the year, the participants also carried out practical VM training by having conversations with 3 residents 2 - 3 times per week, integrated in their daily work. The residents were chosen by each nurse in line with the supervisor's instructions. Conversations with one resident per nurse were videotaped once a month. During the monthly training sessions, the participants viewed these videotapes together with the supervisor, who gave individual feedback. The authors had no influence on running the programme or selecting the participants or residents involved.

\subsection{Participating Nurses}

The study included eight nurses who had completed the entire programme: one registered nurse, four licensed practical nurses, and three nurses' aides. Their median age was 49.5 years (range 33 - 63) and they had a median of 16.5 years (range 1 - 36) of care experience and a median of 6.5 years (range 1 - 36) of experience in the study setting. Five of these eight nurses had been trained in 
dementia care through different short courses.

\subsection{Residents Involved}

Eleven residents ( 9 female, 2 male) with a median age of 85.5 years (range 77 - 94) were involved in the videotaped conversations. Two of these residents had severe visual impairment and one had severe hearing impairment. As assessed with the MDS Cognitive Performance Scale (CPS), these 11 residents had moderate (CPS 3) to very severe (CPS 6) cognitive impairment [13,14], meaning that they had memory problems as well as difficulties finishing thoughts, expressing concrete requests regarding basic needs, making themselves understood by others (e.g. due to problems finding the right word), and making daily decisions. A very severe cognitive impairment (CPS 6) means that a resident is also totally dependent on nurses' assistance for eating.

\subsection{Data Material}

The data consisted of videotaped conversations from the participants' VM training, with at least five months between the first and last recording. The videotapes were recorded by the participants for reflection and supervision during the programme. Initially, one resident per nurse was involved in the videotaped conversations. During the one-year programme, three of these residents died and one did not continue in the VM training as the nurse considered that it was not working. Under the guidance of the certified supervisor, three nurses chose other residents well-known to them and one nurse chose a resident already involved. Thus four of the nurses had conversations with more than one resident during the programme.

For the data analysis, approximately three hours of videotapes were viewed. Videotapes from the beginning of the programme lasted 3 - 14 minutes and videotapes from the end 5 - 36 minutes.

\subsection{Data Analysis}

The basic model for analysis of visual data as described by Collier and Collier [15] was used as a structure for the analysis, conducted in three steps: observing the data as a whole, performing a structured analysis, and returning to the data as a whole. Methods previously used in similar types of analysis were also employed to some extent [16, 17].

Step 1: observing the data as a whole. Initially, all videotapes were viewed by all authors in order to confirm whether the VM was used. Two skilled persons with long experience of VM assisted during this preview. Thereafter the videotapes were viewed repeatedly in order to grasp the content and to inspire ideas for conducting a qualitative analysis guided by the study aim. The steps of the analysis are illustrated in Figure 1.

Step 2: performing a structured analysis. While watching the videotapes, two separate texts (texts 1 and 2) were created for each videotape. Text 1 consisted of notes covering general impressions of communication, such as who initiated the conversational topics and how the nurses interacted with the residents. The notes also included a brief assessment of the nurses' attunement in their communication with the residents, information on the location, in which the videotapes were recorded, and whether any interruptions or distractions occurred during the conversation. Text 2 consisted of transcribed text from the videotapes of nurses' and residents' verbal communication and notes about some non-verbal communication. In order to ensure that full attention was paid to non-verbal communication, at times some videotapes were reviewed with the sound turned off. Particular note was made of eye contact, touch, head nods, gestures, and body posture and orientation. Even though the possibility of studying non-verbal communication is an advantage when using videotapes, it must be considered impossible to capture everything that happens in a conversational situation. The videotapes and transcriptions were kept together as a whole in every step of the analysis, and there was a movement back and forth both within and between all videotapes and transcriptions.

Step 3: returning to the data as a whole. In the third step, Texts 1 and 2 from each videotape for each nurse were brought together for the continuing analysis, which consisted of reading the texts and watching the videotapes together [16]. An overall description of all data was formulated for each nurse, describing changes between videotapes from the beginning and from the end of the programme. The overall descriptions were compared according to any changes of nurses' communication in

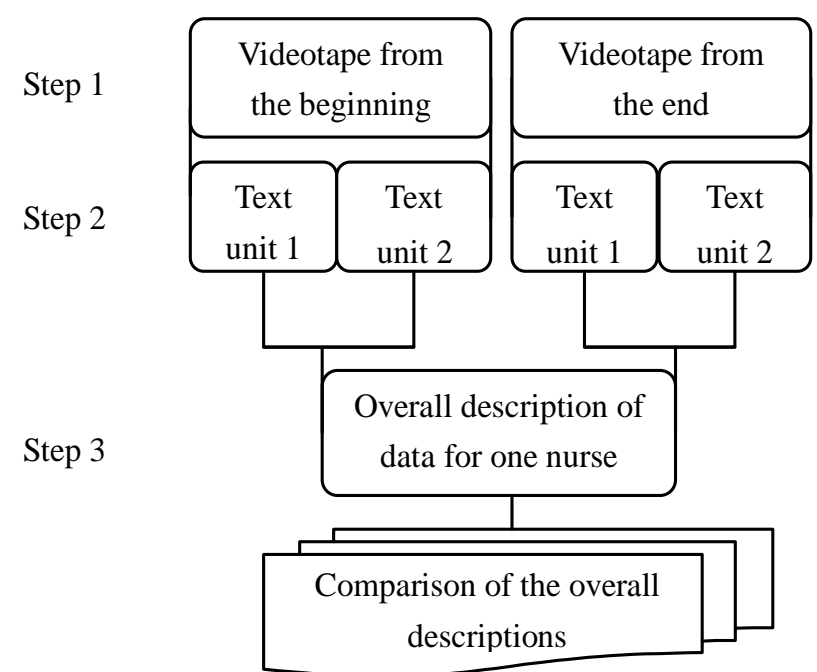

Figure 1. Overview of data analysis Steps 1-3. 
conversations with residents and usage of VM techniques. The videotapes were watched together with reading the overall descriptions. Three sub-patterns were identified: from controlling communication towards developing attentiveness in communication, from ambiguous communication towards developing coherence in communication, and from being open and attentive towards having a refined attuned communication. An overall pattern was also formulated.

To assure trustworthiness of the results, all authors initially viewed the videotapes. During the process of analysis, the videotapes were viewed repeatedly and the authors had continuing discussions to ensure the most reasonable patterns. Moreover, two of the authors (MS, $\mathrm{GH}$ ) watched the videotapes and read the overall descriptions to identify any contradictions regarding the identified patterns (cf. Dahlberg et al. [18]). The authors' pre-understanding may have been affected by previous studies conducted within the larger project that the present study is a part of, but the intention was to be as open-minded as possible to what the videotapes and transcriptions were about.

\subsection{Ethical Considerations}

A pivotal aspect that needs to be considered is the risk of violating the integrity of those being videotaped, which in the present study included both nurses and residents. Residents with DD may forget that they have given informed consent to participate. Therefore, the nurses were instructed to obtain permission from the residents each time they practiced the VM and to stop if the residents showed signs of being uncomfortable. No such incidents seemed to occur. The nurses and the residents' next-ofkin had received oral and written information about the study and were given the opportunity to raise questions about the research. They were informed of their right to discontinue participation without giving any reason, and were guaranteed confidentiality. The next-of-kin signed an informed consent form. At the end of the programme, the nurses could voluntarily hand over their videotapes by their own choice. The videotapes were kept in a locked filing cabinet and not shown to any persons other than the participating nurses and the research team. The Regional Ethical Review Board (No. 04-704/5) approved the study.

\section{RESULTS}

In videotapes from the beginning of the programme, the nurses behaved as if they were unfamiliar with the situation of conversing with residents without specifically being in an everyday care situation that also included doing tasks. Most of the videotapes from this period were recorded in a day room or in the resident's room with the door to the corridor left open. Sometimes there was a radio or television turned on nearby. It was apparent that different nurses showed a variety of communication skills in their conversations with residents. Some nurses communicated seemingly without effort, while others seemed to steer the conversation in a stiffer way with prompting questions that needed to be answered. However, individual nurses developed their skills to varying degrees during the programme. In videotapes from the end of the programme, some nurses seemed to have developed their confidence in having conversations with residents, while others from the beginning exhibited skills indicating an effortless and authentic conversation. Most of the videotaped conversations lasted longer towards the end of the programme, and were recorded in the residents' rooms with no distractions that could be heard or observed.

The comparison of changes in nurses' communication resulted in an overall pattern: nurses' movements within and between various paths when improving their communication skills. This was based on three sub-patterns: from controlling communication towards developing attentiveness in communication, from ambiguous communication towards developing coherence in communication, and from being open and attentive towards having a refined attuned communication. The nurses developed their communication skills within and between these patterns. That is, some nurses developed their skills within the pattern they exhibited from the beginning, while others developed their skills towards another pattern as shown in Table 1.

Table 1. Nurses movements within and between patterns from the start and end of the training programme.

\begin{tabular}{|c|c|c|c|c|}
\hline \multirow{5}{*}{$\begin{array}{l}\text { Start of the } \\
\text { training } \\
\text { programme }\end{array}$} & & \multicolumn{3}{|c|}{ End of the training programme } \\
\hline & & $\begin{array}{l}\text { Towards developing } \\
\text { attentiveness in communication }\end{array}$ & $\begin{array}{c}\text { Towards developing } \\
\text { coherence in communication }\end{array}$ & $\begin{array}{l}\text { Towards having a refined } \\
\text { attuned communication }\end{array}$ \\
\hline & From controlling communication & 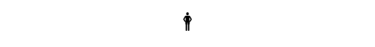 & $\uparrow$ & $\$$ \\
\hline & From ambiguous communication & & $\$$ & $\$$ \\
\hline & From open and attentive communication & & & $\$$ \\
\hline
\end{tabular}

Each figure ( ) in the table represents one nurse. 


\subsection{From Controlling Communication towards Developing Attentiveness in Communication}

In this pattern, the expression "controlling communication" indicates that the communication is steered by the nurse in a prompting way. At the beginning of the programme, nurses took the initiative and initiated topics of conversation. A common topic was "Please tell me what it was like when you were young" or "What did you work with when you were young?" The questions that followed were mostly about facts, even if the residents had spoken about feelings. In one videotape, a resident was talking about experiences from a job and said "It was tough too". The nurse responded: "Were the kids tough? Annoying adults, perhaps?" with the result that the resident said "Time passed quickly" and then was silent.

In these situations, the nurses used a neutral tone of voice and spoke at a fast pace without allowing pauses and without giving the residents enough time to respond. In some situations, the nurses appeared to respond to their own questions or were quick to make comments from their own point of view; for example, one nurse interrupted a resident and said: "It was not so bad, pretty nice...huh". At times when the residents were able to answer more elaborately, the nurses sometimes interrupted by posing questions such as "Who is it?" or "What's his name?" When residents had a hard time answering questions, misunderstandings could arise, as the nurses and residents appeared to be talking about different issues. Sometimes it seemed as if the nurses did not understand the residents; they just said "okay" and let the residents continue talking. One example of a nurse having a hard time following the resident's thoughts occurred when the resident said: "No, I cannot stand him at all" and the nurse, who was not able to follow up, answered: "No, no ... ... yes ... have you watched TV today?” In other situations, it appeared as if the nurses doubted whether what residents said could be true and actually said: "I do not believe that..." During these conversations, the nurses sat at a distance from the resident and physical contact was sparse.

The changes that occurred in the nurses' communication within this pattern were: using a slower pace, allowing some pauses, and showing more attentiveness towards the topics residents talked about. For example, when a resident talked about a tough job experience the nurse responded "In what way was it tough?" and received an elaborate answer from the resident. The use of $\mathrm{VM}$ in conversations with residents consisted primarily of verbal VM techniques such as using nonthreatening factual words ("What do you do about it?"), rephrasing ("You do not want to sit and wait for them"), and remi- niscing ("Have you always helped them?”). Occasionally, imagining the opposite was used, as when asking "Was there any time when everything had gone against you?", or using polarity "What is the best thing about getting older?" Conversations at the end of the programme were also characterized by the nurses' sitting close to the resident, placing a hand on the resident's forearm, and silently waiting for the resident to continue as long as the resident seemed to have the initiative.

To summarize, in conversations at the end of the programme the nurses showed more adherence to the topic of the conversation and generally paid more attention to what the residents were saying. However, it seemed that knowledge of the VM had not been internalised in the communication, as the change in attentiveness was not persistent and towards the end of the conversations the nurses returned to communicating in more or less the same way as at the beginning of the programme.

\subsection{From Ambiguous Communication towards Developing Coherence in Communication}

In conversations within this pattern, the nurses initiated topics, asked questions about facts, responded and commented seemingly from their own points of view, and interrupted when they had difficulties understanding the residents. However, in contrast to this controlling manner in using verbal communication, the nurses talked at a slow, calm pace and in a warm voice. They sat close to the residents, at the same or a lower level, while leaning forward and trying to make eye contact. Physical contact was established with the residents during the entire conversations, usually by the nurse placing a hand on the resident's arm.

The main change in the nurses' communication within this pattern was that a more coherent verbal and nonverbal communication was seen during the conversations at the end of the programme. The communication moved towards more verbal attentiveness to what the residents were talking about. The residents seemed to speak more freely, which for example meant giving longer answers. The same verbal VM techniques as described earlier were used, with the addition of using ambiguity when the residents talked about things that were difficult to understand. For example, when a resident talked about "... nice little white creatures, so they get to see what it is", the nurse rephrased this as a question: "White creatures?" and the resident responded "Yes, you could call them that". During the conversations at the end of the programme, the types of non-verbal communication that could be recognised as VM techniques seemed to be more apparent. These were maintaining genuine close eye contact; using a clear, low, loving tone of voice; 
touching; and observing and matching the person's motions and emotions (mirroring). Mirroring was achieved partly by matching emotions with voice pitch and showing joy through laughter when the residents laughed.

To summarize, in conversations at the end of the programme, more verbal attentiveness was apparent towards residents' communication, which together with non-verbal attentiveness resulted in a more coherent communication.

\subsection{From Being Open and Attentive towards Having a Refined Attuned Communication}

The nurses who exhibited openness and attentiveness at the beginning of the programme were those who let the residents decide what the conversations would be about and when they would end. In these situations, the residents were given time to start talking and to answer questions. The nurses listened openly to the residents without relating to what they themselves thought or felt, even if the topics the residents were talking about were unknown and sometimes incomprehensible to them. In situations where residents got upset or talked about sad topics, the nurses sometimes seemed to ease the residents' sadness. In one conversation, a resident was talking about the ending of life and said "But now time has run out and so." First the nurse asked "Mm. ... Does it feel that way?" and the resident responded "Well, you know that it is so." After a short pause the nurse said "....It is spring soon ... so everything feels a little easier." The resident continued "Yes, but right now it feels hard, I think." The nurse then asked "What is the worst?" and invited the resident to continue talking about her feelings. During these conversations, the nurses' tone of voice was warm and they sat close to the residents, on the same or at a lower level, leaning forward and sometimes touching the residents.

The changes of nurses' communications within this pattern and the differences from the other patterns can be described by the expression "refined attunement". This implies that the nurses were using the skills necessary for an attentive communication in which the residents could see, hear, and feel the nurses during conversations. At the end of the programme, the nurses seemed more confident with just listening to what the residents said and adjusting themselves to the residents' ability to communicate in ways that could include talking, singing, or humming. The conversations included several verbal and non-verbal communication methods that could be identified as VM techniques. In one conversation, a nurse and a resident communicated mostly by singing. When the nurse started singing, the resident started humming, and continued even though the nurse stopped singing. This was repeated a few times. When the nurse finally thanked the resident for their shared time and their singing to- gether, the resident smiled and looked as if she had enjoyed the moment.

Even if issues came up that upset the residents, the nurses remained attuned. In one conversation, a resident spoke about her fear of dying. The nurse listened attentively, closely followed what the resident was talking about, and used methods that could be identified as VM techniques: rephrasing, ("No, you do not want to die"), using nonthreatening factual words ("What is it that is frightening?"), using polarity ("What is the worst thing about feeling this way?"), reminiscing ("Have you always been afraid of death?”), and using ambiguity with rephrasing (when the resident, coining a new phrase, said "There will certainly be a porridge mess", the nurse rephrased this: "It would be a porridge mess"). Through the whole conversation the nurse sat close to the resident, leaning forward, maintaining genuine, close eye contact, using a clear, low, loving tone of voice, and touching. The nurse was holding the resident's hands during the entire conversation, which lasted for about 30 minutes. Towards the end of the conversation the resident said “...let us leave death for now ... I think life is wonderful anyway”.

To summarize, nurses who seemed to have developed a refined attuned communication had probably internalised knowledge that allowed them to make use of the VM in a highly sensitive way.

\section{DISCUSSION}

The aim of the present study was to explore any changes in nurses' communication skills with residents with DD when using the VM, as observed in videotaped conversations. Three sub-patterns formed the overall pattern: nurses' movements within and between various paths when improving their communication skills.

All participating nurses developed their communication skills, although to different degrees as described in the three sub-patterns. The movement within and between the sub-patterns could indicate that the nurses in one way or another had internalised knowledge of the VM. It is reasonable to assume that the development of skills could be related to the nurses' varied personal prerequisites and abilities at the start of the programme. This also applies to the time frame needed to change the habitual way of working, which may vary between nurses, as well as how to handle the strain caused by a process of change. Although a refined attuned communication seemed almost self-evident for some of the nurses, others seemed to need more training and practice. Another reason for the various patterns observed in the conversations could be that some nurses' actions were influenced by their awareness that their conversations were being recorded. However, previous studies have reported that participants 
become accustomed to the presence of the video camera, and act in the same way as they usually do $[16,17,19]$. In our study, the analysis of videotaped conversations was considered an appropriate method for studying changes in communication, since the conversation situation as a whole was included. The conversations analysed in the present study were planned as part of the participants' VM training, and were therefore not conducted during everyday care. Moreover, the focus was on the nurses' communication, even though conversations between residents and nurses are interpersonal. The fact that the participating nurses chose which training sessions to videotape and conducted the video recordings themselves could be seen as both an advantage and a disadvantage. For residents, it could be an advantage not to be confronted with the presence of an unknown person during the conversation situations. This also had an ethical dimension, in that the presence of such a person could have caused harm by creating unnecessary anxiety, which also might have influenced the results.

Insufficient knowledge about DD and how it affects communication abilities can pose a risk that residents' communication is devalued or ignored [3]. It may not only be residents' communication abilities that are devalued; nurses may also devalue the support they themselves can offer [20]. This might have affected nurses' communication within the first sub-pattern, and could be an example of when personal prerequisites and abilities affect communication skills. Furthermore, the nurses' feelings of uncertainty in communicating with residents and also how to use the VM might hinder nurses from being open-minded to the world of the resident and listening attentively. It seemed as if it was not always easy or even possible for the nurses to understand what the residents talked about. However, the nurses appeared to find it easier to follow the conversations and the content if the residents were allowed to have enough time (and hence opportunity) to continue talking about what was on their mind at the time.

Richer conversations took place within the second sub-pattern, which could be interpreted as meaning that the residents responded more to the nurses' non-verbal than to their verbal communication. Hubbard et al. [21] and Bucks and Radford [22] have shown that in spite of DD, residents' ability to use and interpret non-verbal communication was relatively well preserved. This highlights the importance of offering nurses training in interpreting non-verbal communication [23]. Within this pattern, it was apparent that touch and closeness seemed to facilitate communication; similar results have previously been reported [21]. However, not all residents are comfortable with closeness and touch, and for some nurses it can be too demanding; hence, closeness and touch are not suitable to all [7].
The refined, attuned communication in the third subpattern appeared when the nurses were open-minded toward the residents' expressions of their experiences and listened attentively. This could be understood as meaning that the nurses were authentically present. Zyblock [24] states that presence as a way of being with a resident in an authentic relationship promotes mutual respect, honesty, and dignity. Attentive listening is the foundation of this presence [25], and is considered to be more than just hearing the words (cf. Shipley [26]). Attentive listening requires silencing one's own thoughts and focusing solely on the narrator [27]. According to Klaver and Bart [28], attentiveness in caring can create an intersubjective space that can help to bring about caring relationships. In the present study, refined attunement included attentiveness to what the residents seemed to want to talk about, to how they expressed themselves, to the content of the conversations, to their tone of voice and pace, and to their gestures and body movements. This approach could also be likened to care from a life-world perspective, which emphasizes an open attitude and sensitivity towards a person's experiences and expressions [29]. Attempting to understand the meaning of a person's present activity or conversation requires the listener to set aside their own criteria of truth and reality, even if what the speaker is expressing seems strange or unreal [30]. Body language and mood or emotional attunement can give a powerful message of the meaning of a situation [29]. This implies that a life-world perspective could be helpful for nurses when trying to understand residents' communication.

The results from the present study are in line with nurses' experiences of the VM as described in interviews [7]. The observations from the videotapes could be considered to validate the results of the interviews. Previous studies have shown that VM-trained nurses improved their responses to the needs and behaviours expressed by residents [10]. The present results are also in line with research showing that communication training with different intervention components can develop nurses' communication skills $[5,6]$. It is therefore reasonable to suggest that similar results could be achieved with other training programmes similar to the VM programme; this needs to be studied further. There is also a need for studies of VM-trained nurses' communications that take place during everyday care, and of residents' communications.

\section{CONCLUSION}

All nurses developed their communication skills during the VM training, albeit to different degrees. Lack of knowledge about DD and how it affects a resident's communication abilities might affect the possibility of 
successful communication with residents. The ability to have a congruent verbal and non-verbal communication -that is, being present and listening attentively, and being close to and touching the resident-seemed to promote closer relationships. The results in the present study are in congruence with the experiences previously described by the participants [7]. It is reasonable to believe that the programme contributed to improving the nurses' skills in communicating with residents with DD, as they also held in the interviews.

\section{RELEVANCE TO CLINICAL PRACTICE}

Care of residents with DD requires skilful communication, and a VM training programme could give nurses the possibility to develop their skills. This programme should include both theoretical and practical training with individual supervision. The key elements of such a programme include knowledge about DD and how it affects communication abilities, as well as knowledge of how to be present and listen attentively.

\section{ACKNOWLEDGEMENTS}

We are very grateful to the nurses and residents in the nursing home wards who participated in this study; and to Kristina Telerud and EvaLena Gustafsson, who assisted during the initial data analysis.

The study was supported by Ersta Sköndal University College and financed by grants from AFA Insurance.

\section{REFERENCES}

[1] Balalle, C., Jayalath, D., Shankar, K. and Ashaye, K. (2010) Prevalence and management of behavioural and psychiatric symptoms on a continuing care unit for patients with dementia. International Journal of Psychiatry in Medicine, 4, 425-438. doi:10.2190/PM.40.4.f

[2] Edberg, A., Bird, M., Richards, D.A., Woods, R., Keeley, P. and Davis-Quarrell, V. (2008) Strain in nursing care of people with dementia: Nurses' experience in Australia, Sweden and United Kingdom. Aging \& Mental Health, 2, 236-243. doi:10.1080/13607860701616374

[3] Cohen-Mansfield, J. and Parpura-Gill, A. (2008) Practice style in the nursing home: Dimensions for assessment and quality improvement. International Journal of Geriatric Psychiatry, 4, 376-386. doi:10.1002/gps.1888

[4] Ward, R., Vass, A.A., Aggarwal, N., Garfield, C. and Cybyk, B. (2008) A different story: Exploring patterns of communication in residential dementia care. Ageing and Society, 5, 629-651 doi:10.1017/S0144686X07006927

[5] McGilton, K.S., Boscart, V., Fox, M., Sidani, S., Rochon, E. and Sorin-Peters, R. (2009) A systematic review of the effectiveness of communication interventions for health care providers caring for patients in residential care settings. Worldviews on Evidence-Based Nursing, 3, 149159. doi:10.1111/j.1741-6787.2009.00155.x
[6] Vasse, E., Vernooij-Dassen, M., Spijker, A., Rikkert, M. O. and Koopmans, R. (2010) A systematic review of communication strategies for people with dementia in residential and nursing homes. International Psychogeriatrics, 2, 189-200. doi:10.1017/S1041610209990615

[7] Söderlund, M., Norberg, A. and Hansebo, G. (2012) Implementation of the validation method: Nurses' descriptions of caring relationships with residents with dementia disease. Dementia, 5, 569-587. doi:10.1177/1471301211421225

[8] Feil, N. (2002) The validation breakthrough: Simple techniques for communicating with people with "Alzheimer's-type dementia”. Health Professions Press, Baltimore.

[9] Neal, M. and Briggs, M. (2003) Validation therapy for dementia. The Cochrane Database of Systematic Reviews, Article ID: CD 001394. doi:10.1002/14651858.CD001394

[10] Fine, J.I. and Rouse-Bane, S. (1995) Using validation techniques to improve communication with cognitively impaired older adults. Journal of Gerontological Nursing, 6, 39-45.

[11] Finnema, E., Dröes, R., Ettema, T., Ooms, M., Adèr, H., Ribbe, M. and van Tilburg, W. (2005) The effect of integrated emotion-oriented care versus usual care on elderly persons with dementia in the nursing home and on nursing assistants: A randomized clinical trial. International Journal of Geriatric Psychiatry, 4, 330-343. doi:10.1002/gps.1286

[12] The Validation Training Institute, Inc. (2011) http://www.vfvalidation.org/web.php?request=index

[13] Hawes, C., Morris, J.N., Phillips, C.D., Mor, V., Fries, B.E. and Nonemaker S. (1995) Reliability estimates for the minimum data set for nursing home resident assessment and care screening (MDS). The Gerontologist, 2, 172-178. doi:10.1093/geront/35.2.172

[14] Hansebo, G., Kihlgren, M., Ljunggren, G. and Winblad, B. (1998) Staff views on the Resident Assessment Instrument, RAI/MDS, in nursing homes, and the use of the Cognitive Performance Scale, CPS, in different levels of care in Stockholm, Sweden. Journal of Advanced Nursing, 3, 642-653. doi:10.1046/j.1365-2648.1998.00707.x

[15] Collier, J. and Collier, M. (1986) Visual anthropology: Photography as a research method. University of New Mexico Press, Albuquerque.

[16] Hansebo, G. and Kihlgren, M. (2002) Carers' interactions with patients suffering from severe dementia: A difficult balance to facilitate mutual togetherness. Journal of Clinical Nursing, 2, 225-236. doi:10.1046/j.1365-2702.2002.00601.x

[17] Skovdahl, K., Larsson Kihlgren, A. and Kihlgren, M. (2003) Dementia and aggressiveness: Video recorded morning care from different care units. Journal of Clinical Nursing, 6, 888-898. doi:10.1046/j.1365-2702.2003.00809.x

[18] Dahlberg, K., Dahlberg, H. and Nyström, M. (2008) Reflective lifeworld research. Studentlitteratur, Lund.

[19] Latvala, E., Vuokila-Oikkonen, P. and Janhonen, S. (2000) 
Videotaped recording as a method of participant observation in psychiatric nursing research. Journal of Advanced Nursing, 5, 1252-1257. doi:10.1046/j.1365-2648.2000.01383.x

[20] Hansebo, G. and Kihlgren, M. (2000) Patient life stories and current situation as told by carers in nursing home wards. Clinical Nursing Research, 3, 260-279. doi:10.1177/10547730022158582

[21] Hubbard, G., Cook, A., Tester, S. and Downs, M. (2002) Beyond words: Older people with dementia using and interpreting nonverbal behaviour. Journal of Aging Studies, 2, 155-167. doi:10.1016/S0890-4065(02)00041-5

[22] Bucks, R.S. and Radford, S.A. (2004) Emotion processing in Alzheimer's disease. Aging and Mental Health, 3, 222-232. doi:10.1080/13607860410001669750

[23] Magai, C., Cohen, C.I. and Gomberg, D. (2002) Impact of training dementia caregivers in sensitivity to nonverbal emotion signals. International Psychogeriatrics, 1, 25-38. doi:10.1017/S1041610202008256

[24] Zyblock, D.M. (2010) Nursing presence in contemporary nursing practice. Nursing Forum, 2, 120-124. doi:10.1111/j.1744-6198.2010.00173.x

\section{APPENDIX 1}

Description of VM techniques (according to Feil [8]).

\section{Before Starting}

Centring: Focusing on one's own breathing, closing out emotions like anger and frustration, in order to be able to listen empathically to what the person with DD is communicating.

\section{Verbal VM Techniques}

Using nonthreatening factual words to build trust:

Using the words "who", "what”, "when”, “where”, and "how" in questions, but avoiding the word "why".

Rephrasing: Using the same key words and matching the person's tone of voice, rhythm, and feeling.

Using polarity: Asking questions about what is the worst, best, most, or least.

Imagining the opposite: Asking if there are times when the situation doesn't happen, to be used with rephrasing and reminiscing.

Reminiscing: Helping to retrieve familiar coping skills of handling stress.

Using ambiguity: Using non-specific pronouns as a substitute for expressions or non-dictionary words that are hard to understand.

Identifying and using the preferred sense: Building trust by using words that match a person's language and
[25] Bunkers, S.S. (2010) The power and possibility in listening. Nursing Science Quarterly, 1, 22-27. $\underline{\text { doi:10.1177/0894318409353805 }}$

[26] Shipley, S.D. (2010) Listening: A concept analysis. Nursing Forum, 2, 125-134. doi:10.1111/j.1744-6198.2010.00174.x

[27] Hessel, J.A. (2009) Presence in nursing practice: A concept analysis. Holistic Nursing Practice, 5, 276-281. doi:10.1097/HNP.0b013e3181b66cb5.

[28] Klaver, K. and Baart, A. (2011) Attentiveness in care: Towards a theoretical framework. Nursing Ethics, 5, 686693. doi:10.1177/0969733011408052

[29] Todres, L., Galvin, K. and Dahlberg, K. (2007) Life-worldled healthcare: Revisiting a humanising philosophy that integrates emerging trends. Medicine, Health Care, and Philosophy, 1, 53-63. doi:10.1007/s11019-006-9012-8

[30] Ashworth, A. and Ashworth, P. (2003) The lifeworld as phenomenon and as research heuristic, exemplified by a study of the lifeworld of a person suffering Alzheimer's disease. Journal of Phenomenological Psychology, 2, 179205. doi:10.1163/156916203322847137

preferred sense (e.g. vision, hearing, sensory).

\section{Non-Verbal VM Techniques}

Maintaining genuine, close eye contact: Eye contact can be threatening or stimulating, and should be used sensitively.

Using a clear, low, loving tone of voice: The tone of voice can convey a warm atmosphere and respect. Strong emotions expressed with an emotional tone should be matched.

Observing and matching the person's motions and emotions (mirroring): Observing and matching motions and emotions can help caregivers to enter the emotional world of persons with DD.

Touching: Touching should be based on individual needs of body contact, ranging from friendly touching on the forearm to being very close by for those who need close body contact.

Using music: Using music and singing familiar songs can be used as a way of communication when the ability to express oneself with words has declined.

\section{VM Techniques with a Therapeutic Approach}

Linking behaviour to the unmet human need: Basic human needs that are difficult to express (such as emotions) can instead be expressed as repetitive movements or as symbols. 